\title{
Evaluación y control del riesgo químico por vapores orgánicos en los despachadores de combustible de una estación de servicio
}

\section{(Assessment and control of chemical risk from organic vapors for attendants in a gas station)}

\author{
Stephanie Ehmig Santillán ${ }^{1}$
}

\begin{abstract}
Resumen:
El trabajo de investigación se fundamenta en el monitoreo, evaluación y recomendaciones para el control del riesgo químico producido por vapores orgánicos (benceno, tolueno y xileno) procedentes del combustible (gasolina súper y extra), al que están expuestos los despachadores de combustible de una estación de servicio. Dada la concentración medida de los vapores orgánicos (benceno, tolueno y xileno), el riesgo químico al que están expuestos los despachadores de combustible es aceptable en el área de abastecimiento o despacho de combustible. Las recomendaciones constituyen medidas de control enfocadas a reforzar la prevención para preservar la salud y bienestar de este grupo de trabajadores y evitar la manifestación de enfermedades ocupacionales a mediano y largo plazo.
\end{abstract}

Palabras clave: monitoreo; evaluación; riesgo químico; enfermedades ocupacionales; medidas de control.

\begin{abstract}
:
This research comprises monitoring, assessment and recommendations for chemical risk originating from organic vapors (benzene, toluene and xylene) of fuel (super and extra gasoline) to which attendants at a gas station are exposed. Given the concentration measured of organic vapors (benzene, toluene and xylene) the chemical risk to which attendants are exposed in the supply area is acceptable. Control measures are recommended to ensure healthy working conditions for gas station attendants and also to avoid occurrence of occupational diseases in the medium or long term.
\end{abstract}

Keywords: monitoring; assessment; chemical risk; occupational diseases; control measures.

\footnotetext{
${ }^{1}$ EP PETROECUADOR, Quito - Ecuador (stephanie.ehmig@eppetroecuador.ec)
} 


\section{Introducción}

Existen emisiones de vapores orgánicos provenientes del combustible durante su despacho a los vehículos livianos en una estación de servicio. Su capacidad de almacenamiento de combustible es de 130.200 galones, de los cuales aproximadamente son despachados 45.000 galones diarios. Actualmente en dicha estación de servicio trabajan 43 despachadores de combustible que están expuestos a vapores orgánicos durante las 8 horas diarias de su jornada laboral.

Según la concentración de vapores orgánicos a la que estén expuestos los despachadores de combustible, podrían manifestarse afectaciones en su salud, mediante efectos agudos (corta duración y se presentan enseguida) o crónicos (larga duración y se presentan paulatinamente) pudiendo desencadenar en una enfermedad ocupacional en el trabajador o hasta en su deceso.

En tal virtud, La principal motivación para emprender esta investigación fue la incertidumbre respecto a la concentración de los vapores orgánicos benceno, tolueno y xileno a la que se encuentran expuestos los despachadores de combustible de una estación de servicio durante su jornada laboral. Considerando que al menos el benceno es un agente químico clasificado como categoría A1 carcinógeno humano confirmado tanto por American Conference of Governmental Industrial Hygientists, así como por International Agency for Research on Cancer of the World Health Organization (World Health Organization, 2011) (Tufts University, 2001). Además si bien existen varios factores que inciden en la manifestación de efectos agudos o crónicos además de la exposición a estos vapores orgánicos, es importante determinar el riesgo químico de exposición para así poder controlarlo y prevenir cualquier consecuencia indeseable.

En tal virtud, se realizó la medición de la concentración de vapores orgánicos, específicamente de benceno, tolueno y xileno, a la que se encuentran expuestos los despachadores de combustible durante el tiempo que dura su jornada laboral (8 horas); se efectuó la evaluación del riesgo químico de exposición de estos vapores orgánicos en los despachadores de combustible para determinar si es alto, medio o bajo; se establecieron medidas de control, con recomendaciones coherentes con el riesgo químico de exposición determinado, que estén encaminadas a la prevención de efectos agudos o crónicos en la salud de los despachadores de combustible por exposición a los vapores orgánicos benceno, tolueno y xileno. De esta manera se pretende controlar y disminuir el riesgo químico en los despachadores de combustible por dichos vapores orgánicos.

La investigación pretendió comprobar que el de riesgo químico de exposición a vapores orgánicos, específicamente de benceno, tolueno y xileno en los despachadores de combustible es seguro y consecuentemente evita la manifestación de efectos agudos o crónicos en su salud.

El estudio consistió en desarrollar información primaria y recabar información secundaria de diversas fuentes bibliográficas inherentes al tema de estudio. La información primaria se 
fundamentó en el monitoreo de benceno, tolueno y xileno con monitores personales pasivos 3M 3500, análisis de laboratorio, cálculo de las concentraciones, tratamiento estadístico de resultados (Castejón, 1994) y evaluación del riesgo a través de la comparación con los límites de exposición respectivos (Bartual Sánchez, y otros, 1989).

Del total de despachadores de combustible (43) fue seleccionada una muestra denominada grupo homogéneo de exposición (9). El tiempo de muestreo correspondió a la duración de una jornada laboral completa (8 horas).

Si bien en 8 de 9 muestras, los resultados demostraron un riesgo aceptable en el área de abastecimiento o despacho de combustible, 1 muestra excede el límite de exposición profesional para benceno, esto quiere decir que probablemente en otras áreas de la estación de servicio existen concentraciones de benceno superiores al límite de exposición establecido para una jornada laboral de 8 horas.

\section{Metodología}

Sujetos: La existencia de varias personas que realizan tareas similares en condiciones ambientales parecidas, plantea la posibilidad de realizar mediciones de la exposición a una parte de ellas y ahorrar recursos, considerando así una sola exposición común para todas. A esta parte se le denomina Grupo Homogéneo de Exposición (GHE) (Castejón, 1994). La norma UNE-EN 689 (Comité Técnico CEN/TC, 1995) recomienda tomar un mínimo de 1 trabajador por cada 10, quien constituirá el GHE. En este caso se tomaron 2 trabajadores por cada 10.

Técnicas e instrumentos: para obtener información primaria se efectuó el monitoreo de benceno, tolueno y xileno con monitores personales pasivos 3M 3500. Este es un dispositivo de muestreo diseñado para medir una concentración promedio de ciertos contaminantes sobre un intervalo de tiempo medido. Cuando es utilizado como un monitor personal debe ser ubicado cerca de la zona de respiración del individuo expuesto a ambientes potencialmente peligrosos. (3M OHES Division, 2003) El monitor 3M 3500 contiene una sola almohadilla de carbón adsorbente que sirve para captar un amplio rango de vapores orgánicos ${ }^{2}$, tiene un peso de $9 \mathrm{~g}$ por lo que es liviano, pequeño y no interfiere con las actividades del trabajador. No necesita de baterías, bombas o equipos de calibración. La figura 1 muestra una imagen del monitor pasivo 3M 3500:

\footnotetext{
2 “3M Technical Data Bulletin, Organic Vapor Monitor, Sampling and Analysis Guide”. (s.f.). Recuperado, de http://multimedia.3m.com/mws/media/1107310/organic-vapor-monitor-sampling-and-analysis- guide.pdf
} 


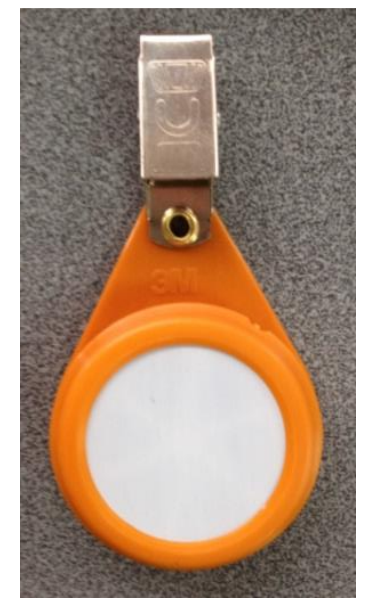

Figura 1. Monitor pasivo 3M 3500

Una vez efectuado el monitoreo se enviaron las muestras al laboratorio para realizar el análisis respectivo. Con los resultados del laboratorio se calculó la concentración de cada contaminante (benceno, tolueno y xileno) mediante la aplicación de varias fórmulas (Castejón, 1994). Asimismo se les aplicó a los resultados un tratamiento estadístico para así poder evaluar el riesgo con la comparación de los límites de exposición respectivos (TLV-TWA y VLA-ED) (Huici, y otros, 1999).

La técnica utilizada para recabar información secundaria fue la técnica de recopilación de información y análisis de documentos.

Para registrar la información observada en campo se utilizaron los siguientes instrumentos:

- Formularios de monitoreo

- Cadenas de custodia

- Cámara fotográfica

- Filmadora

Procedimiento: la formulación del problema es la incertidumbre de la concentración de los vapores orgánicos (benceno, tolueno y xileno) ${ }^{3}$ a la que están expuestos los despachadores de combustible en la estación de servicio Amazonas de EP Petroecuador durante su jornada laboral. En tal virtud se requiere medir la concentración de vapores orgánicos, específicamente de benceno, tolueno y xileno, a la que se encuentran expuestos los despachadores de combustible el tiempo que dura su jornada laboral (8 horas); evaluar si el riesgo químico de exposición a estos vapores orgánicos en los despachadores de combustible es aceptable; establecer las medidas de control, mediante recomendaciones coherentes con el riesgo químico de exposición determinado, que estén encaminadas a la prevención de efectos agudos o crónicos en la salud de los despachadores de combustible por exposición a dichos vapores orgánicos. De esta manera se

\footnotetext{
3 "Benceno -Efectos en la salud". (1997)."Tolueno - Efectos en la salud". (1997). "Xileno - Efectos en la salud”. (1997). Recuperado, de http://www.ccsso.ca/oshanswers/chemicals/chem_profiles/
} 
procura controlar y disminuir el riesgo químico en los despachadores de combustible por vapores orgánicos del benceno, tolueno y xileno.

Se realizó un análisis de factores de riesgo mediante la identificación de las características físicoquímicas del agente, condiciones del puesto de trabajo y comportamiento de los individuos. Además se realizó la evaluación de la exposición a agentes químicos que consistió en estimar la magnitud del riesgo y sus características mediante las siguientes etapas: identificación de posibles exposiciones; determinación de factores ligados al puesto de trabajo; valoración inicial y evaluación detallada.

Para llevar a cabo el monitoreo, que fue considerado la parte crucial de la investigación, se recabó bastante información sobre los condicionantes tecnológicos y estratégicos que definieron el agente, el cómo, dónde y cuándo medir. De tal forma que se definieron como agentes al benceno, tolueno y xileno; el sistema e instrumental para la toma de muestras fue a través del sistema de captación por difusión (pasiva) (Bartual, 1982) (Guardino, y otros, 2001) mediante los monitores pasivos 3M 3500; la ubicación de los monitores fue en el área respiratoria del trabajador (en la solapa del overol, lo más cerca posible del cuello); el tiempo de monitoreo fue Tipo A que equivale a la toma de una muestra de duración igual al período de exposición, es decir las 8 horas de una jornada laboral completa; el número de muestras se aplicó al grupo homogéneo de exposición, es decir a 9 despachadores de combustible; el criterio para seleccionar los trabajadores sujetos al monitoreo fue según las islas de despacho con mayor movimiento; la técnica analítica utilizada por el laboratorio fue la cromatografía de gases-FID.

Una vez obtenidos los resultados del laboratorio, se procedió a aplicar las fórmulas para el cálculo de las concentraciones de los contaminantes por cada muestra tomada, incluyendo aquellas consideraciones (Martí Veciana, 2000) (Martí, 2000) establecidas para el efecto. Posteriormente se aplicó un tratamiento estadístico a los datos y la evaluación del riesgo mediante la comparación con los límites de exposición respectivos (TLV-TWA y VLA-ED).

\section{Resultados}

En las tablas 1 y 2 se ilustra el riesgo químico de exposición de los despachadores de combustible a benceno, tolueno y xileno en el área de abastecimiento o despacho de combustible, el cual es bajo, debido a que su concentración está muy por debajo de los límites establecidos para el efecto. 
Tabla 1. Concentración de BTX y comparación con VLA-ED

\begin{tabular}{|c|c|c|c|}
\hline $\begin{array}{c}\text { Código de } \\
\text { Muestra }\end{array}$ & $\begin{array}{c}\text { C8 Benceno } \\
\left(\mathbf{m g} / \mathbf{m}^{\mathbf{3}}\right)\end{array}$ & $\begin{array}{c}\text { C8 Tolueno } \\
\left(\mathbf{m g} / \mathbf{m}^{\mathbf{3}}\right)\end{array}$ & $\begin{array}{c}\text { C8 Xileno } \\
\left(\mathbf{m g} / \mathbf{m}^{\mathbf{3}}\right)\end{array}$ \\
\hline M1-i7 & 0,0123521 & 0,0243200 & 1,3122322 \\
\hline M2-i1 & 0,0373697 & 0,1226283 & 0,6818735 \\
\hline M3-i9 & 8,8505818 & 23,5110730 & 8,9433919 \\
\hline M4-i8 & 0,0619541 & 0,0968103 & 0,6847317 \\
\hline M5-i4 & 0,0226614 & 0,0330503 & 0,6769036 \\
\hline M6-i7 & 0,0086504 & 0,0141931 & 1,3128262 \\
\hline M7-i1 & 0,0117645 & 0,0135457 & 1,3155900 \\
\hline M8-i9 & 0,0101637 & 0,0132019 & 1,3496874 \\
\hline M9-i8 & 0,0278759 & 0,0304915 & 0,6790506 \\
\hline $\begin{array}{c}\text { VLA-ED } \\
\left(\mathbf{m g} / \mathbf{m}^{\mathbf{3}}\right)\end{array}$ & $\mathbf{3 , 2 5}$ & $\mathbf{1 9 2}$ & $\mathbf{2 2 1}$ \\
(INSHT, 2014) & & & \\
\hline
\end{tabular}

Tabla 2. Concentración de BTX y comparación con TLV-TWA

\begin{tabular}{|c|c|c|c|}
\hline $\begin{array}{c}\text { Código de } \\
\text { Muestra }\end{array}$ & $\begin{array}{c}\text { C8 Benceno } \\
(\mathrm{ppm})\end{array}$ & $\begin{array}{c}\text { C8 Tolueno } \\
(\mathrm{ppm})\end{array}$ & $\begin{array}{c}\text { C8 Xileno } \\
(\mathrm{ppm})\end{array}$ \\
\hline M1-i7 & 0,0038633 & 0,0064624 & 0,3026022 \\
\hline M2-i1 & 0,0116880 & 0,0325852 & 0,1572408 \\
\hline M3-i9 & 2,7681607 & 6,2474392 & 2,0623559 \\
\hline M4-i8 & 0,0193771 & 0,0257247 & 0,1578999 \\
\hline M5-i4 & 0,0070877 & 0,0087822 & 0,1560947 \\
\hline M6-i7 & 0,0027055 & 0,0037714 & 0,3027392 \\
\hline M7-i1 & 0,0036795 & 0,0035994 & 0,3033765 \\
\hline M8-i9 & 0,0031789 & 0,0035081 & 0,3112394 \\
\hline M9-i8 & 0,0087186 & 0,0081023 & 0,1565898 \\
\hline $\begin{array}{c}\text { TLV-TWA } \\
\text { (ppm) } \\
\text { (ACGIH, 2005) }\end{array}$ & $\mathbf{0 , 5}$ & $\mathbf{5 0}$ & $\mathbf{1 0 0}$ \\
\hline
\end{tabular}

Se verificó que el riesgo químico de exposición de los despachadores de combustible a los vapores orgánicos benceno, tolueno y xileno, es seguro en el área de abastecimiento o despacho de combustible, lo cual evitaría la manifestación de efectos agudos o crónicos en su salud.

A pesar de que en el monitoreo realizado al individuo codificado como M3-i9, el contaminante benceno excede tanto el valor límite ambiental de exposición diaria (VLA-ED) como el valor límite umbral - concentración promedio ponderada para 8 horas (TLV-TWA). Es importante señalar que el individuo no se mantuvo en su lugar de trabajo asignado para el día de muestreo (isla de despacho \# 9), debido a que en reiteradas ocasiones estuvo en el interior del área administrativa (oficinas), cuadrando caja, almorzando, conversando con otros compañeros de trabajo, en definitiva estuvo entrando y saliendo del área administrativa. Este resultado plantea la hipótesis de que posiblemente haya mayor concentración de benceno inclusive en valores superiores a los límites establecidos para el efecto en el área administrativa, especialmente debido a la migración de contaminantes por el comportamiento de las corrientes aéreas, factores de dispersión en el aire y velocidad del viento. 
El resultado final de la desviación estándar geométrica, calculada sin las concentraciones de benceno, tolueno y xileno del monitor codificado como M3-i9, determina que las demás concentraciones de benceno, tolueno y xileno expresan una dispersión de distribución logarítmiconormal (Luna Mendaza, 2000) por debajo de la dispersión máxima de valores y que los valores son similares y precisos.

A pesar de que las concentraciones de benceno, tolueno y xileno incluyen su valor de incertidumbre, tampoco superan los valores límites ambientales de exposición diaria (VLA-ED) ni los valores límites umbral - concentración promedio ponderada para 8 horas (TLV-TWA), a excepción de la muestra codificada como M3-i9 únicamente en el contaminante benceno.

Las concentraciones de los contaminante benceno, tolueno y xileno no son iguales en las mismas islas de despacho monitoreadas en diferentes días, corroborando así la afirmación de (Luna, 2000) "Es un hecho comprobado que la concentración ambiental en un puesto de trabajo varía de forma aleatoria a lo largo de la jornada laboral y de una jornada a otra".

De conformidad con la afirmación de (Luna Mendaza, 1998) "A menudo no es posible conocer la verdadera concentración media existente en un puesto de trabajo a causa de las múltiples variaciones que sufre la concentración ambiental a lo largo de la jornada laboral y entre diferentes jornadas"; no obstante, en función del valor de la media geométrica (gc), el valor más probable de la media de las concentraciones se acerca a $0,0115620 \mathrm{mg} / \mathrm{m}^{3}$.

De acuerdo con la recomendación de (3M España, 1999) "Los valores TLV deben utilizarse como directrices o recomendaciones para el control de riesgos potenciales para la salud. No están pensados para ser utilizados como estándares legales", los valores límite umbral (TLV) fueron utilizados únicamente como directrices para el control de riesgos potenciales para la salud.

El tiempo de monitoreo de los vapores orgánicos benceno, tolueno y xileno al que se encuentran expuestos los despachadores de combustible fue de 463 - 477 minutos, es decir una duración equivalente a un rango del $96.5 \%$ al $99.4 \%$ del turno de trabajo o de la jornada laboral (mínimo $25 \%$ o 120 minutos).

Si bien el 56\% del GHE trabaja de 3 a 6 años y apenas el 22\% trabaja más de 6 años en la estación de servicio en el puesto de trabajo despachador de combustible, están expuestos a un riesgo químico aceptable en el área de abastecimiento o despacho de combustible, no obstante se desconoce la concentración de estos vapores a la que estén expuestos los demás trabajadores en las áreas: administrativa, de almacenamiento de combustible, de descarga de combustible para auto-tanques y de acceso. 
Debido a que el riesgo químico por exposición a vapores orgánicos en los despachadores en el área de abastecimiento o despacho de combustible es aceptable, no es necesario implementar medidas de control, sin embargo se proponen varias medidas de control en la fuente y en el receptor que podrían ser adoptadas para reforzar la prevención.

\section{Discusión}

De conformidad con estudios similares al presente trabajo de investigación, realizados a partir del año 2000, las concentraciones de (benceno, tolueno y xileno) están por debajo de los límites de exposición respectivos para el puesto de trabajo de despachador de combustible en estaciones de servicio que expenden combustibles.

Hubo un estudio en donde se realizaron monitoreos en ciertas estaciones climáticas del año y en diferentes años, debido a que desde el año 2000 se emitieron regulaciones en países europeos y sudamericanos que limitaron el contenido de benceno en las gasolinas. En este estudio, se determinó que a mayor temperatura mayor volumen de vapores orgánicos y mayor concentración de benceno, pero a pesar de ello las concentraciones se mantuvieron por debajo de los límites de exposición profesional.

Cabe mencionar que en el presente estudio, los monitoreos fueron realizados a temperaturas moderadas $17^{\circ} \mathrm{C}-18^{\circ} \mathrm{C}$.

Las ventajas de este estudio fueron principalmente:

- El acceso y apertura para realizar la investigación en la estación de servicio.

- Colaboración para la provisión de recursos tecnológicos, así como el servicio de análisis en el laboratorio.

Respecto al aporte personal realizado fue minucioso y significativo para poder llevar a cabo el monitoreo y posteriormente el análisis e interpretación de resultados.

\section{Conclusiones y Recomendaciones}

El riesgo químico en los despachadores de combustible por exposición a los vapores orgánicos del combustible benceno, tolueno y xileno en el área de abastecimiento o despacho de combustible en el tiempo que dura su jornada laboral (8 horas) diarias es aceptable y seguro.

La concentración medida de los vapores orgánicos benceno, tolueno y xileno está muy por debajo de los límites establecidos para el efecto, lo cual evitaría la manifestación de efectos agudos o crónicos en la salud de los despachadores, corroborando así que la hipótesis planteada en la presente tesis es certera.

Debido a que el riesgo químico por exposición a vapores orgánicos en los despachadores en el 
área de abastecimiento o despacho de combustible es aceptable, no es necesario implementar medidas de control, sin embargo a continuación se proponen varias medidas de control en la fuente y en el receptor que podrían ser adoptadas para reforzar la prevención.

Incorporar nuevas tecnologías para minimizar la exposición delos despachadores de combustible a vapores orgánicos, como dotara las mangueras de suministro del surtidor de un ajuste flexible y hermético a la boca del depósito de combustible del vehículo, con una doble tubuladura, una que conduzca el combustible al tanque del vehículo mientras que la otra recoja los vapores orgánicos emanados durante la carga de combustible, de esta forma el cierre hermético impedirá que se escapen los vapores orgánicos a la atmósfera y sean reconducidos nuevamente al mismo tanque de almacenamiento de combustible. Esta medida contribuirá significativamente en la reducción de las concentraciones de benceno, tolueno y xileno.

Sugerir la implementación de políticas de Estado, como limitar el contenido de benceno a un máximo de $1 \%(\mathrm{v} / \mathrm{v})$, mediante importantes modificaciones legales en lo que respecta a la calidad de los combustibles que se expenden a nivel nacional.

Realizar monitoreos en las demás áreas de la estación de servicio Amazonas, tales como: administrativa, almacenamiento de combustible, descarga de combustible para auto-tanques y de acceso.

Considerar la alternativa de optar por el abastecimiento de combustible en los vehículos, mediante el autoservicio.

Capacitar a todo el personal de la estación de servicio sobre los diferentes tipos de riesgos a los que se encuentran expuestos en sus puestos de trabajo.

Continuar sometiendo a los trabajadores de la estación de servicio a los exámenes médicos ocupacionales periódicos. Incluir la realización de exámenes especiales como la medición de indicadores biológicos a través de metabolitos con la determinación del ácido SPMA en orina.

Realizar indagaciones asociadas a hábitos, actividades y trabajos efectuados por los despachadores de combustible fuera de su jornada laboral en la estación de servicio.

Introducir la realización de pausas activas laborales a todo el personal de la estación de servicio a fin de incrementar su productividad.

Considerar la alternativa de un cambio en la modalidad de trabajo definida para los despachadores de combustible de 10 días de trabajo x 5 días de descanso a $5 \times 2$. 


\section{Bibliografía}

3M España. (abril de 1999). 3M. Recuperado el 14 de febrero de 2015, de multimedia.3m.com: http://multimedia.3m.com/mws/media/3310490/monitor- badge.pdf?fn=MonitorES.pdf

3M OHES Division. (2003). 3M. Recuperado el 20 de febrero de 2015, de www.multimedia.3m.com: http://multimedia.3m.com/mws/media/211130O/air- monitoringguide-3500-3510-3520-3530-3550-3551.pdf

ACGIH. (2005). Secretaría del Trabajo y Previsión Social, Estados Unidos Mexicanos. Recuperado el 26 de febrero de 2015, de www.stps.gob.mx: http://www.stps.gob.mx/DGIFT_STPS/PDF/2005TLVsBEIsofACGIHHandbook.pdf

Bartual Sánchez, J., \& Guardino Solá, X. (1989). Instituto Nacional de Seguridad e Higiene en el Trabajo. Recuperado el 25 de febrero de 2015, de www.insht.es:

http://www.insht.es/InshtWeb/Contenidos/Documentacion/FichasTecnicas/NTP/Fichero s/201a300/ntp_244.pdf

Bartual, J. (1982). Instituto Nacional de Seguridad e Higiene en el Trabajo. Recuperado el 13 de febrero de 2015, de www.insht.es:

http://www.insht.es/InshtWeb/Contenidos/Documentacion/FichasTecnicas/NTP/Fichero s/101a200/ntp_151.pdf

Castejón Vilella, E. (1994). Instituto Nacional de Seguridad e Higiene en el Trabajo. Recuperado el 21 de febrero de 2015, de www.insht.es:

http://www.insht.es/InshtWeb/Contenidos/Documentacion/FichasTecnicas/NTP/Fichero s/301a400/ntp_347.pdf

Comité Técnico CEN/TC. (1995). Instituto Técnico de Prevención Seguridad e Higiene Industrial.

Recuperado el 2 de marzo de 2015, de www.itpshi.es:

http://www.itpshi.es/documents/jornada_silicio/UNE_EN_689.pdf

Guardino, X., \& Ramos, J. (2001). Instituto Nacional de Seguridad e Higiene en el Trabajo. Recuperado el 12 de febrero de 2015, de www.insht.es: http://www.insht.es/InshtWeb/Contenidos/Documentacion/FichasTecnicas/NTP/Fichero s/501a600/ntp_587.pdf

Huici, A., \& Ferrer, R. (1999). Instituto Nacional de Seguridad e Higiene en el Trabajo. Recuperado el 26 de febrero de 2015, de www.insht.es: http://www.insht.es/InshtWeb/Contenidos/Documentacion/FichasTecnicas/NTP/Fichero s/501a600/ntp_526.pdf 
INSHT. (2014). Instituto Nacional de Seguridad e Higiene en el Trabajo. Recuperado el 27 de febrero de 2015, de www.insht.es:

http://www.insht.es/InshtWeb/Contenidos/Documentacion/LEP\%20_VALORES\%20LIMITE/Valor es\%20limite/Limites2014/FINAL\%20-\%20Web\%20v5\%20-\%20LEP\%202014\%20-\%2029-012014.pdf

Luna Mendaza, P. (2000). Instituto Nacional de Seguridad e Higiene en el Trabajo. Recuperado el 20 de febrero de 2015, de www.insht.es: http://www.insht.es/InshtWeb/Contenidos/Documentacion/FichasTecnicas/NTP/Fichero s/501a600/ntp_554.pdf

Luna Mendaza, P. (2000). Instituto Nacional de Seguridad e Higiene en el Trabajo. Recuperado el 13 de febrero de 2015, de www.insht.es: http://www.insht.es/InshtWeb/Contenidos/Documentacion/FichasTecnicas/NTP/Fichero s/501a600/ntp_553.pdf

Luna, P. (1998). Instituto Nacional de Seguridad e Higiene en el Trabajo. Recuperado el 22 de febrero de 2015, de www.insht.es: http://www.insht.es/InshtWeb/Contenidos/Documentacion/FichasTecnicas/NTP/Fichero s/401a500/ntp_406.pdf

Martí Veciana, A. (2000). Instituto Nacional de Seguridad e Higiene en el Trabajo. Recuperado el 14 de febrero de 2015, de www.insht.es:

http://www.insht.es/InshtWeb/Contenidos/Documentacion/FichasTecnicas/NTP/Fichero s/501a600/ntp_548.pdf

Martí, A. (2000). Instituto Nacional de Seguridad e Higiene en el Trabajo. Recuperado el 14 de febrero de 2015, de www.insht.es:

http://www.insht.es/InshtWeb/Contenidos/Documentacion/FichasTecnicas/NTP/Fichero s/501a600/ntp_547.pdf

Tufts University. (Abril de 2001). Tufts University, Department of Public and Environmental Safety. Recuperado el 26 de Abril de 2015, de www.publicsafety.tufts.edu:

http://publicsafety.tufts.edu/ehs/files/carcinogens.pdf

World Health Organization. (2011). International Agency for Research on Cancer. Recuperado el 26 de Abril de 2015, de www.monographs.iarc.fr:

http://monographs.iarc.fr/ENG/Classification/List_of_Classifications_Vol1-113.pdf 